\title{
THE DUST LANES OF THE BARRED GALAXY NGC 5383
}

\author{
H.B. ANN AND J.M. KIM \\ Pusan National University \\ Dept. of Earth Sciences, Pusan National University, Pusan \\ 609-735, Korea
}

\section{Introduction}

NGC 5383 is a barred galaxy of type ( $\left.\mathrm{R}^{\prime}\right) \mathrm{SB}(\mathrm{rs}) \mathrm{b}$ : pec with well developed dust lanes which provide valuable information about the dynamics of the galaxy. It is well known that dust lanes in barred spiral galaxies represent the loci of shock fronts which are caused by the gas inflow driven by bar potential (Athanassoula 1992). However, detailed morphology of dust lanes depends on the distribution of masses in bulge, disk, and bar as well as bar pattern speed. We conducted a $U B V R I$ and $H_{\alpha}$ surface photometry and SPH simulations for NGC 5383 to investigate the relation between the morphology of dust lanes and the dynamical properties of barred galaxies.

\section{Morphology of dust lanes}

The dust lanes of NGC 5383 show typical characteristics of barred galaxies with strong bar. The dust lane in the western part of the bar is more pronounced than that of the eastern bar. The dust lanes run nearly parallel to the bar axis with offsets of $\sim 10^{\prime \prime}$ from the major axis of the bar toward the leading edge of the bar. They smoothly joined into the inner curved dust lanes which run parallel to the nuclear spiral arms. They are best seen in the B-band images and almost invisible in the I-band.

\section{SPH simulations}

We made SPH simulations to understand the formation and evolution of the dust lanes in NGC 5383. We assumed exponential disk, Plummer spherical potential for bulge, logarithmic potential for dark halo, and bi-axial potential (Long \& Murali 1992) for bar component. The total mass and radius 


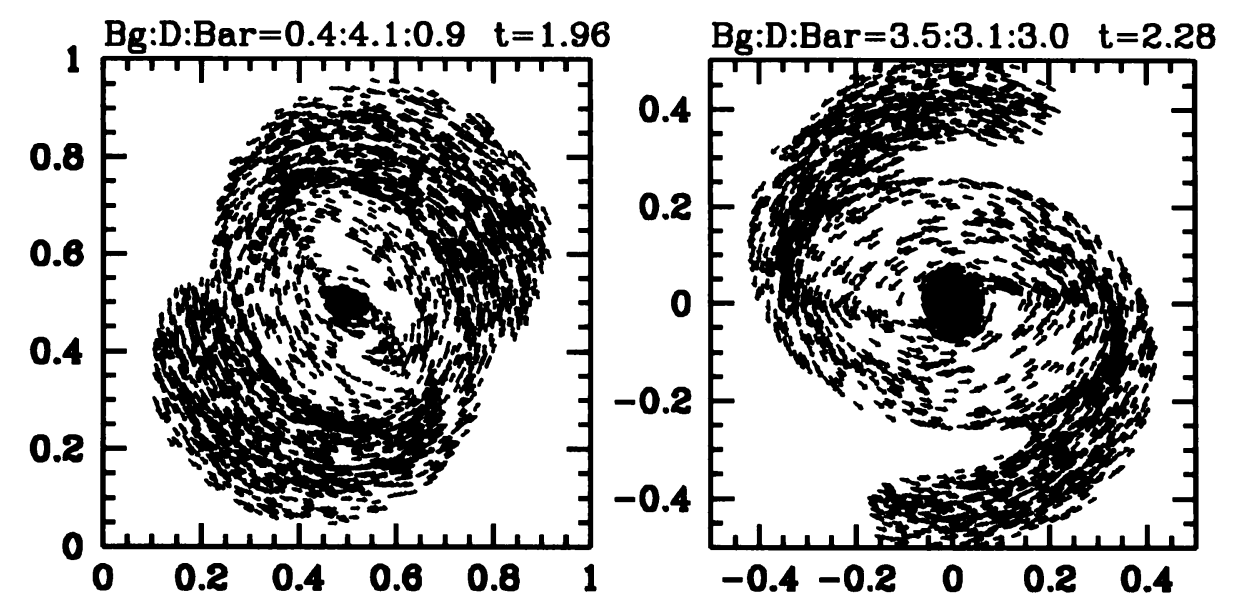

Figure 1. Distribution of gas velocities in models with small bulge (left) and comparable masses in each component (right).

of model galaxies are assumed to be $2 \times 10^{11} M_{\odot}$ and $20 \mathrm{kpc}$, respectively with $\Omega=(10 \sim 100) k m / s e c / k p c$.

\section{Results and discussion}

The shape of dust lanes largely depends on the fraction of bulge mass and bar pattern speed. The straight dust lanes are well developed in the models with small bulges and models with comparable masses in each component. But in models with massive bulges, dust lanes are highly curved. Dust lanes with low pattern speed also have curved shapes.

Dust lanes formed in comparable mass models become very weak after several dynamical time scales $\left(\sim 10^{9} \mathrm{yrs}\right)$. The gas left over after dissolution of dust lanes populates the $\mathrm{x} 2$ orbit and make the oval structure similar to the nuclear region of NGC 5383 .

\section{Acknowledgements}

This work was supported in part by the Basic Science Research Program, Ministry of Education, BSRI-96-5411.

\section{6. references}

Athanassoula, E. 1992, $M N, \mathbf{2 5 9}, 345$

Long, K., and Murali, C. 1992, ApJ, 397, 44 